\title{
The underlying mechanisms of bilateral cerebral remodeling and acupuncture for rehabilitation after post-stroke hemiplegia: study protocol for a cross- sectional multimodal fMRI trial
}

\section{Hualei Geng}

Beijing University of Chinese Medicine Affiliated Dongzhimen Hospital https://orcid.org/0000-00015843-714X

\section{Yue Wang}

Beijing University of Chinese Medicine

\section{Yahui Wang}

Beijing University of Chinese Medicine

\section{Lan Jiang}

Peking Union Medical College Hospital

Pei Chen

$$
\text { Capital Medical University }
$$

\section{Mengxin Lu}

Beijing University of Chinese Medicine

\section{Zhongming Du}

Beijing University of Chinese Medicine

\section{Chen Chen}

Beijing University of Chinese Medicine

\section{Kang Wu}

Beijing University of Chinese Medicine

\section{Lixin Tang}

Beijing University of Chinese Medicine

Zhongjian Tan

Beijing University of Chinese Medicine

\section{Kuangshi Li}

Beijing University of Chinese Medicine

Yihuai Zou ( $\nabla$ zouyihuai2004@163.com )

Beijing University of Chinese Medicine Affiliated Dongzhimen Hospital https://orcid.org/0000-00024003-8244 


\section{Study protocol}

Keywords: Post-stroke, Hemiplegia, Functional magenetic resonance imaging, Functional connectivity, Acupuncture, Bilateral cerebral remodeling

Posted Date: March 8th, 2021

DOI: https://doi.org/10.21203/rs.3.rs-61605/v1

License: (c) (i) This work is licensed under a Creative Commons Attribution 4.0 International License.

Read Full License 


\section{Abstract \\ Background}

Acupuncture plays a positive role in promoting the rehabilitation of post-stroke hemiplegia, but its specific central effect mechanism remains unclear. The changes of cerebral integrity, bilateral regulation and coordination are closer to the actual running state of brain after injury, while the research on bilateral cerebral reconstruction after unilateral motor pathway injury is not sufficient. The study of cerebral functional remodeling after stroke and mechanisms of acupuncture for post-stroke hemiplegia still faces many challenges. This study will explore the the underlying mechanisms of bilateral cerebral remodeling and acupuncture for post-stroke hemiplegia rehabilitation.

\section{Methods}

This is a single-center, cross-sectional, multimodal functional magnetic resonance imaging(fMRI) study, with participants and data analyst blinded. 24 post-stroke patients with motor dysfunction and 24 healthy subjects that meet the inclusion criteria will be recruited in this study. All the patients enrolled in this study will receive the conventional standard medical care according to their chief doctors. Clinical data including motor function scales, high-sensitivity C-reactive protein(hs-CRP), other related scales as well as fMRI imaging data will be collected at baseline and 2 weeks later. Functional connectivity $(\mathrm{FC})$ is the primary and core outcome indicator of this study. Voxel-mirrored homotopic connectivity (VMHC), fractional anisotropy (FA),voxel-based morphometry (VBM), Fugl-Meyer Assessment(FMA) and hs-CRP are secondary outcome indicators. During the MRI scan, true or sham Yanglingquan(GB34) acupuncture stimulation on the left side and passive finger movement will be performed on the participants. The connection between cerebral imaging indicators and clinical indicators will be obtained by calculating the Pearson's correlation coefficient.

\section{Discussion}

The results of our study will help to understand the cerebral remodeling mechanism of motor function improvement after stroke better and provide data support for the better application of acupuncture in clinical practice.

\section{Trial registration:}

Chinese Clinical Trial Registry (ChiCTR1900022220). Regisered on 30 March 2019. http://www.chictr.org.cn/showprojen.aspx?proj=37359

\section{Background}


Stroke is the third leading cause of death of non-communicable diseases in the world [1], while the leading cause of death in China [2]. Motor dysfunction after stroke is the main factor that reduces the quality of life, brings terrible pain to patients, and causes great economic loss to the society.

Rehabilitation is the primary way to promote motor function recovery by evidence-based medicine $[3,4]$, and it is recommended to apply rehabilitation treatment to patients as early as possible [5-7].

Acupuncture, as an important part of traditional Chinese medicine(TCM), has been widely used in the rehabilitation treatment of stroke. WHO has included rehabilitation after stroke in the scope of application that may benefit from acupuncture treatment [8]. Basic studies have shown that acupuncture plays a role in promoting stroke rehabilitation mainly by improving the nerve regeneration of central nervous system and cell amplification, regulating blood flow in ischemic foci, and resisting cell apoptosis $[9,10]$. To explore the mechanism of acupuncture for post-stroke rehabilitation is of great significance for improving the clinical promotion and clinical effect of acupuncture.

When brain completes one-side motion task, the contralateral motor pathway is mainly activated. After stroke, the injury not only occurred in the ipsilateral side, but also in the undamaged contralateral cortex, suggesting that stroke rehabilitation requires the remodeling and reorganization of bilateral hemispheric functions [11]. In the early stage of cerebral infarction, the activation of the affected hemisphere decreases first, followed by the increased activation of the healthy hemisphere, the compensatory recovery of motor function, and the associated motion and other wrong motion models, resulting in excessive hemispheric inhibition on the affected hemisphere and adversely affecting the recovery of motor function $[12,13]$. The motor cortex, motor pathways and cerebral functional connectivity all show bilateral changes after unilateral motor pathway is damaged in cerebral infarction. The contralateral hemisphere plays an important role in stroke rehabilitation. The connectivity of the contralateral hemisphere can be enhanced by the movement of the affected limb, and can reflect the activity status more accurately than the connectivity of the lesion hemisphere [14]. Based on the integral changes of the brain after cerebral infarction and the influence of unilateral cerebral hemisphere injury on bilateral hemispheric synergy, there is still a large space to study the mechanism of acupuncture effect on rehabilitation after stroke.

Magnetic resonance imaging (MRI) -based techniques have excellent spatial resolution, allowing our understandings of cortical remodeling to extend from the basic conceptual level to the level of anatomical and neurobiological mechanisms [15]. The application of multimodal MRI to study the multimodal characterization of ischemic brain diseases has become a hot research topic, mainly including structural MRI and functional MRI(fMRI) of resting- and task-state. The combined use of multimodal technology plays an important role in the imaging characterization of different modes during the occurrence and development of stroke, as well as the early diagnosis, therapeutic effect and prognosis assessment of stroke [16].

Based on the above background and viewpoints, the aims of this study are to: (1) Focusing on the problems of bilateral cerebral regulation and coordination, corticospinal tract (CST) and primary motor 
cortex (M1) will be used as the main research spots to describe the characteristics of brain injury after post-stroke hemiplegia and the mechanisms of brain functional remodeling in rehabilitation. (2) The comparison between true and sham acupoints of Yanglingquan(GB34), patients and healthy subjects will be set to explore the similarities and differences of the central effects of acupoints and non-acupoints from the perspective of bilateral cerebral regulation. (3) Supported by multimodal imaging technology, the characteristics of passive movement and changes before and after acupuncture in bilateral major motor brain areas will be expressed. The mechanisms of acupuncture effect in rehabilitation of post-stroke hemiplegia will be investigated by multi-dimensional and combined methods.

\section{Methods}

\section{Study design}

This is a single-center, cross-sectional, multimodal fMRI study to explore the underlying mechanisms of bilateral cerebral remodeling and acupuncture for rehabilitation after post-stroke hemiplegia. 24 poststroke patients with motor dysfunction and 24 healthy subjects will be recruited in this study. Clinical data including motor function scales, hs-CRP, and other related measurements, and fMRI imaging data will be collected at baseline and 2 weeks later. During the MRI scan, true or sham Yanglingquan(GB34) acupuncture stimulation on the left side and passive finger movement will be performed on the participants. (Fig. 1)

Detailed flowchart of this study is showed in Fig. 1

\section{Participants recruitment}

This study will be carried out at Dongzhimen Hospital Affiliated to Beijing University of Chinese Medicine from April 2019 to August 2022. We will screen and enroll the patients who meet the inclusion criteria from Department of Neurology, Rehabilitation and Acupuncture by Electronic Medical Record. We will include eligible healthy subjects by putting up posters, distributing leaflets, etc. When potential participants are willing to join the study, they or their immediate family members will sign an informed consent before allocation which explains all the information they need to comprehend, including the purpose and procedures of this study, obligations and rights of participants, benefits and risks and right to quit at any time without conditions. Participants will receive a signed and dated copy of the informed consent. The protocol will be reported following Standard Protocol Items: Recommendations for Interventional Trials (SPIRIT) statement (Additional file 1).

The detailed schedule of the study procedures is displayed in Fig. 2

\section{Participants}

\section{Inclusion criteria}




\section{Inclusion criteria for patients' group}

Any patients enrolled must meet all the following criteria: (1) patients with ischemic stroke who conform to the diagnostic criteria[17] and whose course of disease is within 1.5 months;(2) right-handed;(3) aged from 40 to 75 years old;(4) patients with left hemiplegia, the infarct is located in the right basal ganglia and/or the corona radiate region;(5)no conscious disorder and the condition is relatively stable;(6) no psychotropic medications having been taken within 1 month;(7) no metal in the body and no contraindication of nuclear magnetic resonance examination;(8) informed consent must be signed by the patient self or his/her immediate family member.

\section{Inclusion criteria for healthy subjects' group}

Any participants enrolled must meet all the following criteria: (1) right-handed;(2) aged from 40 to 75 years old;(3) proved to be health by physical examination(without organic or significant functional diseases);(4) no family history of mental or neuron system inheritance;(5) no experience for joining research similar to this experiment;(6) no physical abnormalities (such as cold, headache, cough, etc.) during the experimental period;(7) no excitatory medications have been taken within 2 months;(8) no metal in the body and no contraindication of nuclear magnetic resonance examination;(9) informed consent must be signed by the subject self.

\section{Exclusion criteria}

\section{Exclusion criteria for patients' group}

Patients will be excluded if they match any of the following criteria: (1)combining with psychiatric disorders or any other serious primary diseases;(2)being pregnant, lactating or menstrual;(3)severe skull anatomical asymmetry or definite lesions being found by MRI scans;(4)taking part in similar neuroimaging test within 1 month.

\section{Exclusion criteria for healthy subjects' group}

Subjects will be excluded if they match any of the following criteria: (1)being menstrual, pregnant or lactating;(2)severe skull anatomical asymmetry or definite lesions being found by MRI scans.

\section{Randomization and blinding}

In this study, we will enroll 24 patients and 24 healthy subjects. All the 48 participants will receive fMRI scans twice. During the first scan, 12 patients and 12 healthy subjects will receive the true Yanglingquan(GB34) stimulation, and rest of the participants will receive sham Yanglingquan(GB34) stimulation. At the second scan, participants will receive the opposite acupoint stimulation from the first. The sequence of acupuncturing true or sham Yanglingquan(GB34) will be arranged according to a random table. The random sequence of first scan will be generated by an independent professional statistician who is not involved in the study with the software SPSS 20.0. The random sequence will be sealed in an opaque envelope and kept by a person who is not involved in recruitment. The acupuncturist 
will be told the sequence before fMRI scan due to the natural characteristic of acupuncture. The clinical outcome assessors, fMRI scanner, data analysts and participants will be blinded to the sequence. The study will be unblinded by principal investigator after all the study complished.

\section{Sample size}

In the literature on the study of sample of acupuncture-neuroimaging mechanism, it is emphasized that the sample size range of 16-32 cases can ensure sufficient statistical efficacy when the samples are of high homogeneity [18]. This study chooses the stroke patients with hemiplegia within 1.5 month, and requires that the infarction lesions are located on the unilateral motion pathway (right side of basal ganglia and/or corona radiate region). The course and lesions of the disease are of high homogeneity. Combining image analysis and statistical analysis to the technical requirement of the sample, we plan to recruit 24 participants in patients' group and the healthy subjects' group respectively.

\section{Participant and public involvement}

Participants in this study will not be involved in the procedures of design, recruitment or any other conduction of the trial. The patients and their chief doctors will be informed of the results of the fMRI to help make a better therapeutic regimen. Members of the research group will explain the fMRI scanning results to healthy subjects.

\section{INTERVENTIONS}

\section{Acupuncture at Yanglingquan(GB34) during the MRI scanning}

All participants will undergo fMRI scans of acupoint stimulation at true or sham Yanglingquan(GB34) acupoint once respectively, with a 2-week time interval. Whether true or sham acupoints for the first scan will be arranged according to a random table.

All the needles used in the study are disposable sterile silver needles(specification parameter: $\varphi 0.40 \times$ $40 \mathrm{~mm}$ ) purchased from Beijing Zhongyantaihe Medical Instrument Co., LTD., and manufactured by Suzhou Shenlong Medical Instrument Co., LTD..

Acupoint selection is performed according to the National standard GB/T 12346-2006 Name and Location of Acupoints. Yanglingquan (GB34) is located on the outside of the lower leg, in the middle of the concave of the anterior and inferior of the fibula head. Sham acupoint is located in Yanglingquan(GB34) horizontal outward 1 cun.

After skin disinfection routinely, a sterile silver needle for single use will be inserted vertically for 1.5-2 cun at the left acupoint with inforcing-reducing manual stimulation at the frequency of $1 \mathrm{~Hz}$. At the end of each scan, the amount of stimulus will be evaluated by Needle Sensation Assessment. 
Due to the position and dark environmen during MRI scans, participants will not be aware of that whether true or sham acupoint they are being stimulated.

\section{Passive finger movement during the MRI scanning}

Passive finger movement is set as the basic motion background of the task-state. The same professional acupuncturist will conduct the passive finger movement task which is operated by letting the left thumb tap the left index finger at a frequency of $1 \mathrm{~Hz}$ to the subjects [19].

\section{Conventional treatment}

All the patients enrolled in this study will receive the conventional standard medical care according to their doctors. Researchers of this study will not interfere with the routine treatment of patients.

\section{MEASUREMENTS}

Once the patients are enrolled, we will complete the collection of disease background information first. Then, Fugl-Meyer Assessment(FMA) , hs-CRP level test and the first fMRI scan will be performed. The second MRI scan and FMA will be finished 2 weeks later. Other clinical scales, including National Institutes of Health Stroke Scale (NIHSS), Modified Ashworth Scale(MAS), Brunnstrom Scale(BSS), Modified Rankin Scale(MRS), Motricity Index(MI), Barthel Index(BI), Hamilton Depression Scale(HAMD), Mini-Mental State Examination (MMSE) and Needle Sensation Assessment Scale(NSAS) will also be assessed as additional indicators. Except the motor function scales and hs-CRP, the healthy subjects will receive the same assessments as the patients.

\section{MRI data acquisition}

The fMRI scheme in this study refers to the research conclusions of the Institute of Automation, Chinese Academy of Sciences and adopts a non-repeatable event-related experimental design pattern, which conforms to the basic operational process of acupuncture in the clinical practice and is suitable for the study of dynamic response signals of acupuncturing central nerves.

\section{MRI scanning condition}

The fMRI examination will be performed with a 3.0 Tesla scanner (Siemens, Sonata, Germany) in Dongzhimen Hospital, The First Affiliated Hospital of Beijing University of Chinese Medicine.

Scanning parameters: Functional MRI will be applied with T2×WI gradient echo-planar imaging (EPI) sequence: Repetition time(TR), 2000ms; Echo time(TE), 30ms; Matrix, 128×128; Field of view(Fov), 240×240mm; Slice thickness, 5.0mm; Gap, 0mm; Phase encode direction, A >>P; Flip Angle, 90; Fat suppr, Fat sat. The three-dimensional structure imaging scan of whole brain will be scanned using T1W1 sequence, with a Slice thickness of $1.0 \mathrm{~mm}$; TR/TE, 1900ms/3.93ms; Fov, 240×240 and Matrix, $256 \times 256$.

\section{MRI scanning protocol}

The fMRI scan scheme will be showed in Fig.3 
(1) resting-state BOLD imaging(BOLD fMRI) scan

Before the scan, the subjects will be required to rest in the supine position for 30 minutes. After being completely calm, they will be told to close eyes and wear earplugs to maintain the audiovisual closed state. The Magnetic resonance coil will be fixed, and the head position will be kept still by foam pad. The participants should avoid falling asleep and conducting systematic thinking activities at the same time. After 8 minutes and 10 seconds of continuous scanning, the resting-state scanning will be completed.

(2) Task-state scan: Passive finger movement of the left hand

The improved method of block test will be adopted (resting-task-resting-task-resting-task-resting-taskresting-task-resting) to carry out continuous fMRI scanning. Each block takes 40 seconds(20" for resting, 20 " for task), a total of 5 blocks and a total of 3 minutes and 40 seconds of this step.

(3) Diffusion tensor imaging (DTI)

This step will take 5 minutes and 10 seconds.

(4) Acupuncturing-state scanning

The needle will be inserted into the left Yanglingquan(GB34) point or sham point, then the needle will be retained for 10 seconds, after which the needle will be continuously twisted for 1 minute at a frequency of $1 \mathrm{~Hz}$. At last, the needle will be retained for 8 minutes before released. Continuous fMRI scanning will be performed during the above procedures.

(5) High-resolution anatomical T1-weighted imaging (T1W1)

This step will take 4 minutes and 10 seconds.

\section{Neuroimaging outcomes}

Neuroimaging outcomes in this study are composed of FC, VMHC, FA and VBM, which will be obtained after fMRI scanning and imaging data analysis.

FC is the primary and core outcome indicator of this study. FC can be acquired by using resting-state functional magnetic resonance imaging scans. Several studies have proved that $\mathrm{FC}$ values are positively correlated with the patients' motor function recovery [20-22]. The different FC values in the interhemispheric motor cortex (M1) after stroke can reflect long-term recovery of motor function, which makes FC a predictor of motor function recovery [23]. In this study, we will determine the bilateral cerebral FC values to explore the mechanisms underlying the cerebral remodeling after stroke.

VMHC, FA, VBM are secondary outcome indicators of this study. VMHC can reflect the bilateral functional connections [24]. FA can assess the structural reorganization of white matter tracts quantitatively via 
DTI $[25,26]$. VBM has been widely used to detect gray matter and can quickly and easily explore structural differences between groups at a whole-brain level [27].

\section{Clinical outcome assessments}

Fugl-Meyer Assessment

FMA is one of the secondary outcome indicators of this study. Abundant evidence shows that FMA is highly recommended as a clinical and research tool for evaluating changes in motor dysfunction following stroke $[28,29]$. According to the scoring rules of the scale, the higher the score, the better the motor function.

High-sensitivity C-reactive protein (hs-CRP)

Hs-CRP is a secondary outcome indicator of the study. Hs-CRP is a typical acute-phase protein, widely present in the serum and other body fluids $[30,31]$. It can be used as a kind of serum biochemical marker for the etiological classification and prognosis of ischemic stroke [32].

Additional outcome indicaters

NIHSS is the most widely used scale to assess the neurologic impairment severity of ischemic stroke in clinical practice and research [33]. The MAS and BSS are broadly used as tools to measure spasticity [34]. MRS and BI are used to assess disability after stroke [35]. MI is used for the evaluation of motor functions of the pinch grip, elbow flexion, shoulder abduction, ankle dorsiflexion, knee stretch and hip flexion [36]. The above are the commonly used scales to evaluate the neurological function and motor function of patients after stroke.

Depression increased the risk for poor outcomes of disability and death in patients with stroke, and HAMD is used to assess the emotion of depression [37]. The MMSE is a screening tool to get a general image of cognitive functioning of a patient [38].

The evaluation results of NSAS can be used as a reference index of acupuncture efficacy [39]. The NSAS will be evaluated after each fMRI by the acupuncturist.

\section{Incidence of adverse events}

Any adverse events that may happen in this study, including claustrophobia during fMRI scans and sweating, fainting, dizziness or any other adverse events ascribed to acupuncture must be recorded in detail.

If the adverse events are too severe for the subjects to continue the study, the study must be stopped immediately, with appropriate first aid measures, and reported to the principal investigator at once.

\section{Data management}

Page 10/22 
Clinical data needs to be recorded on a printed case report form(CRF) at every visit point. For each participant, there will be separate CRF. And once the study is finished, the data on CRFs will be entered into the EpiData electronic database by two researchers separately. When inconsistent data appears, the third researcher will check the original printed CRFs to correct the errors.

The MRI scans will be performed by professional technicians. They will ensure the quality of the MRI imaging data. When unqualified data appears, an additional scan will be done. The MRI imaging data will be stored in a dedicated hard drive.

\section{Quality control}

In order to ensure the accuracy and quality of the study, we have set out the researcher's manual, developed a standardized technical scheme, and guaranteed the qualification of the researchers. All researchers must be trained to fully understand this protocol and standard operation procedures before beginning. Acupuncture and passive finger movement will be performed by the same acupuncturist throughout the study. Professional researchers will provide technical guidance and assistance in image processing and data analysis to ensure the accuracy and authority of data analysis.

\section{STATISTICAL ANALYSIS}

\section{Neuroimaging data analysis}

The preprocessed method of T1 image

The structure images will be unified to the AC-PC line with a self-coded word at first. The Freesurfer software package(https://surfer.nmr.mgh.harvard.edu/) will be used to perform the cortical segmentation, peel off the scalp and subcortical parts and distinguish the cortical regions. Finally, the cortical structure images will be obtained. Then, the cortical structure images will be unified to the standard coordinates on the AFNI platform(Analysis of Functional Neurolmages)( https://afni.nimh.nih.gov/)and the preprocessed images will be obtained.

The preprocessed method of EPI image

First, Freesurfer software package will be used to align the cortical structure images unified to standard coordinates with the EPI images, and the alignment matrix will be obtained. Then, the matrix projection will be applied to the EPI images to obtain the EPI images aligned with the cortical structure map. AFNI platform will be used to perform cortex-based Gaussian Blur on the EPI images obtained.

Analysis method for resting-state functional connectivity

The random effect model of the SPM software package (https://www.fil.ion.ucl.ac.uk/spm) will be used to calculate the data of passive finger movement of left hands. Monte Carlo Simulation(MCS) will be used to correct the activation images. Select the highest point of the activation and the voxel of its radius within $10 \mathrm{~mm}$ as seed points, and apply REST software package (REpresentational State Transfer) to 
steady state phase to calculate the seed points and the functional connectivity of the whole brain after acupuncture. Using FDR (False Discovery Rate) for the final correction, get the functional connection map.

Analysis method for VMHC

DPARSF (http://rfmri.org/DPARSF) software will be used to invoke the REST software for VMHC wholebrain analysis of needle-state and resting-state data. For each subject, the VMHC values will be obtained by calculating the Pearson's correlation coefficient between the time series of each individual voxel in one hemisphere and the mirror alleles in the opposite hemisphere. Then Fisher $Z$ conversion will be performed to improve the normal distribution. The obtained value is VMHC value, which can be used for group statistical analysis.

Analysis method for task-state

SPM12 software will be used to process the preprocessed EPI images in random effect model. Twosample one-side $T$ test will be performed on the resting and task brain images respectively to calculate the T value of each pixel. A pixel at a certain significance level will be considered to be a pixel with remarkable significance. After the Results module analysis of SPM12 software, the statistical parameter graph will be obtained. The brain areas of the parameter graph will be identified by a self-programmed software and the results will be displayed by MRIcroN software.

Analysis method for DTI image

AFNI software will be used to combine the DTI image and T1 image to calculate the diffusion tensor, FSL TBSS (FMRIB Software Library) (https://fsl.fmrib.ox.ac.uk/fsl/fslwiki/) software will be used to calculate the FA value of each subject, and then FSL software will be used to make comparison between groups.

Analysis method for grey matter volume

SPM12 software package will be used to segment and register the original images, and all the data will be re-output to the images with voxel of $2 \mathrm{~mm} \times 2 \mathrm{~mm} \times 2 \mathrm{~mm}$. Then Gaussian Blur will be carried out on the obtained gray matter images to get the final preprocessed data, and the blur kernel will be taken as $4 \mathrm{~mm} \times 4 \mathrm{~mm} \times 4 \mathrm{~mm}$. The SPM8 test module will be used to conduct two-sample T test on the VBM diagram between groups, and the volume of voxels in the brain regions of different groups will be quantitatively compared.

\section{Clinical data analysis}

A professional statistician will perform statistical analysis of clinical data using the SPSS 26.0 software package (IBM SPSS $\left.{ }^{8}\right)$. The data will be presented as mean \pm SD. T test analysis, Chi-square test, nonparametric relevant-sample test and non-parametric independent-sample test will be performed according to situations. 
The connection between cerebral imaging indicators and clinical indicators will be obtained by calculating the Pearson's correlation coefficient. All the data, including clinical data and fMRI data will be considered statistically significant with $p$ value $<0.05$.

\section{Discussion}

Cerebral diseases and functional remodeling are important tasks in brain science research [40]. The study of cerebral functional remodeling after stroke still faces many challenges.

According to the Cochrane systematic review, acupuncture may have positive effects on improving the independence of post-stroke convalescence patients and promoting the recovery of overall neurological impairment and specific neurological functions such as movement, cognition and swallowing [41]. A multi-center randomized controlled clinical study of 360 patients showed that the integrated rehabilitation program of TCM and Western medicine including acupuncture could significantly improve the daily living ability of post-stroke patients and other aspects of clinical prognosis compared with conventional western medicine treatment [42]. However, there is still a lack of clinical research on the mechanism of acupuncture promoting the rehabilitation of post-stroke hemiplegia focusing on bilateral cerebral remodeling. This study is expected to provide evidence-based support on this topic.

Yanglingquan(GB34) has been used as the main acupoint selection in scheme of TCM diagnosis and treatment of apoplexy as well as in multi-center clinical research $[42,43]$. In the study of acupuncture mechanism, single acupoint is selected as the intervention method, which has certain advantages for task control and reducing the confounding factors of intervention. The method of extracting sham acupoints by horizontal outward true points in this study is a popular and relatively recognized comparison method at present [44]. In this study, we choose Yanglingquan(GB34) as the test point and its sham point as control.

Previous fMRI studies have shown that stroke not only interferes with the neural network of the lesion, but also affects the distant brain region and the contralateral hemisphere, leading to changes in the functional and effective connections within and between cerebral hemispheres, which are closely related to motion injury and recovery [45]. Therefore, the study of bilateral cerebral remodeling is of great significance to explore the mechanism of rehabilitation of hemiplegia after stroke.

In our previous studies [46-50], we gradually and deeply proved that compared with healthy subjects, acupuncture at Yanglingquan(GB34) in patients with hemiplegia showed specific activation of brain regions and expressed bilateral effects, from the perspectives of single spot, single network and compound network, which provides a relatively deep research foundation for the hypothesis of bilateral cerebral coordinated regulation in this study.

For the purposes of task control and reducing the confounding factors of intervention, single acupoint is selected in this study, which is not the actual clinical practice. 
In summary, this study focuses on the perspective of bilateral regulation and coordination of the brain and explores the mechanisms of bilateral cerebral remodeling and the effect of acupuncture after unilateral motor pathways damage at the functional and structural level. Through multi-modal imaging ( $T 1, \mathrm{DTI}$, resting-state, task-state) and analytical methods ( $F C, V M H C, V B M, F A)$, the observation will be carried out from multiple perspectives. We expect to provide new research ideas for the study of the mechanisms of acupuncture. The visualization method will be used to explore the mechanism of acupuncture effect at the level of integral regulation and coordination of the brain, so as to provide data support for the better application of acupuncture in the treatment of post-stroke hemiplegia.

\section{Trial Status}

The study is in the recruiting phase at present. The first patient was recruited on 19 April 2019, while the first healthy subject was enrolled on 26 October 2019. We are supposed to complete the inclusion of all subjects by September 2022 .

\section{List Of Abbreviations}

hs-CRP, hypersensitive C-reactive protein; FC, functional connectivity; FA, fractional anisotropy; VMHC, Voxel-mirrored homotopic connectivity; VBM, Voxel-Based Morphometry; SP, Stroke Patients; HS, Healthy Subjects; fMRI, functional magnetic resonance imaging; FMA, Fugl-Meyer Assessment; NIHSS, National Institutes of Health Stroke Scale; MAS, Modified Ashworth Scale; BSS, Brunnstrom Scale; MRS, Modified Rankin Scale; BI, Barthel Index; MI, Motricity Index; HAMD, Hamilton Depression Scale; MMSE, MiniMental State Examination; NSAS, Needle Sensation Assessment Scale

\section{Declarations}

Ethics approval and consent to participate

This protocol has been approved by the ethics committee of Dongzhimen Hospital affiliated to Beijing University of Chinese Medicine (NO: DZMEC-KY-2018-58). (Additional file 2) Written, informed consent to participate will be obtained from all participants before enrolled. If the principal investigator is changed during the study and any modification to the clinical study protocol, informed consent, and recruitment materials, the applicant shall submit an application for amendment review.

Consent for publication

Not applicable.

Availability of data and materials

The full protocol will be available from the corresponding author after identification. The results of this study will be published in peer-reviewed publications and disseminated through conferences. 
Competing interests

The authors declare that they have no competing interests.

Funding

This study is supported by The National Natural Science Foundation of China(grant number: 81873257) \Additional file $3 \rrbracket$.The funder has not taken part in the design of the study and collection, analysis, and interpretation of data, writing the manuscript and the decision to publish.

Authors' contributions

HL-G and YW contributed equally to this manuscript. HL-G was responsible for ethical approval and accomplishing the clinical trial registration. The participants are screened and enrolled by $\mathrm{YH}-\mathrm{W}, \mathrm{MX}-\mathrm{L}$ and PC. LJ and CC are in charge of acupuncture stimulation and passive finger movement during the fMRI scan. The clinical data collection and CRF fulfillment are done by ZM-D and KW. Questions related to acupuncture are guided by LX-T. MRI scan is performed by ZJ-T. KS-L is responsible for imaging data processing and analysis. $\mathrm{YH}-\mathrm{Z}$, the corresponding author of this manuscript designed the study and sought funding. All authors read and approved the final manuscript.

Acknowledgements

Not applicable.

\section{References}

1. Collaborators GBDCoD. Global, regional, and national age-sex-specific mortality for 282 causes of death in 195 countries and territories, 1980-2017: a systematic analysis for the Global Burden of Disease Study 2017. Lancet. 2018;392(10159):1736-88. doi:10.1016/S0140-6736(18)32203-7.

2. Zhou M, Wang H, Zeng X, Yin P, Zhu J, Chen W, et al. Mortality, morbidity, and risk factors in China and its provinces, 1990-2017: a systematic analysis for the Global Burden of Disease Study 2017. Lancet. 2019;394(10204):1145-58. doi:10.1016/S0140-6736(19)30427-1.

3. Pollock A, Baer G, Campbell P, Choo PL, Forster A, Morris J, et al. Physical rehabilitation approaches for the recovery of function and mobility following stroke. Cochrane Database Syst Rev. 2014; (4):CD001920. doi:10.1002/14651858.CD001920.pub3.

4. Gittler M, Davis AM. Guidelines for Adult Stroke Rehabilitation and Recovery. JAMA. 2018;319(8):820-1. doi:10.1001/jama.2017.22036.

5. Winstein CJ, Stein J, Arena R, Bates B, Cherney LR, Cramer SC, et al. Guidelines for Adult Stroke Rehabilitation and Recovery: A Guideline for Healthcare Professionals From the American Heart Association/American Stroke Association. Stroke. 2016;47(6):e98-169.

doi:10.1161/STR.0000000000000098. 
6. Chinese Neurology Branch of Medical. Association NG, Neurology Branch, Chinese Medical Association,Cerebrovascular Disease Group, Branch N. Chinese Medical Association,. Chinese guidelines for early rehabilitation treatment of stroke. Chin J Integr Med. 2017;50(6):8. doi:10.3760/cma.j.

7. Neurorehabilitation Group NB, Chinese Medical Association,Cerebrovascular Disease Group, Branch N. Chinese Medical Association,Office of stroke Screening and Prevention Engineering Committee, Ministry of Health. Chinese Guidelines for Stroke Rehabilitation (2011 Edition). Chinese Journal of Frontiers in Medicine (Electronic Edition). 4(6):22. doi: 10.3969/j.

8. NIH Consensus Conference. Acupuncture. JAMA. 1998;280(17):1518-24.

9. Chavez LM, Huang SS, MacDonald I, Lin JG, Lee YC, Chen YH. Mechanisms of Acupuncture Therapy in Ischemic Stroke Rehabilitation: A Literature Review of Basic Studies. Int J Mol Sci. 2017;18(11). doi:10.3390/ijms18112270.

10. Lee GJ, Yin CS, Choi SK, Choi S, Yang JS, Lee H, et al. Acupuncture attenuates extracellular glutamate level in global ischemia model of rat. Neurol Res. 2010;32(Suppl 1):79-83. doi:10.1179/016164109X12537002794200.

11. Heiss WD, Kidwell CS. Advances in stroke: Imaging 2013. Stroke. 2014;45(2):363-4. doi:10.1161/STROKEAHA.113.004102.

12. Stinear CM, Petoe MA, Byblow WD. Primary Motor Cortex Excitability During Recovery After Stroke: Implications for Neuromodulation. Brain Stimul. 2015;8(6):1183-90. doi:10.1016/j.brs.2015.06.015.

13. Dodd KC, Nair VA, Prabhakaran V. Role of the Contralesional vs. Ipsilesional Hemisphere in Stroke Recovery. Front Hum Neurosci. 2017;11:469. doi:10.3389/fnhum.2017.00469.

14. Bajaj S, Housley SN, Wu D, Dhamala M, James GA, Butler AJ. Dominance of the Unaffected Hemisphere Motor Network and Its Role in the Behavior of Chronic Stroke Survivors. Front Hum Neurosci. 2016;10:650. doi:10.3389/fnhum.2016.00650.

15. Grefkes C, Fink GR. Connectivity-based approaches in stroke and recovery of function. Lancet Neurol. 2014;13(2):206-16. doi:10.1016/S1474-4422(13)70264-3.

16. Stinear CM. Prediction of motor recovery after stroke: advances in biomarkers. Lancet Neurol. 2017;16(10):826-36. doi:10.1016/S1474-4422(17)30283-1.

17. Chinese Neurology Branch of Medical Association CDG. Neurology Branch, Chinese Medical Association. Chinese Guidelines for the Diagnosis and Treatment of Acute Ischemic Stroke 2014. Chin J Neurol. 2015;48(4):12. doi:10.3760/cma.j.

18. Friston K. Ten ironic rules for non-statistical reviewers. Neuroimage. 2012;61(4):1300-10. doi:10.1016/j.neuroimage.2012.04.018.

19. Cramer SC, Mark A, Barquist K, Nhan H, Stegbauer KC, Price R, et al. Motor cortex activation is preserved in patients with chronic hemiplegic stroke. Ann Neurol. 2002;52(5):607-16. doi:10.1002/ana.10351.

20. Hordacre B, Goldsworthy MR, Welsby E, Graetz L, Ballinger S, Hillier S. Resting State Functional Connectivity Is Associated With Motor Pathway Integrity and Upper-Limb Behavior in Chronic Stroke. 
Neurorehabil Neural Repair. 2020;34(6):547-57. doi:10.1177/1545968320921824.

21. Li Z, Yang M, Lin Y, Liang S, Liu W, Chen B, et al. Electroacupuncture promotes motor function and functional connectivity in rats with ischemic stroke: an animal resting-state functional magnetic resonance imaging study. Acupunct Med. 2020:964528420920297. doi:10.1177/0964528420920297.

22. Zhang Q, Li J, Huang S, Yang M, Liang S, Liu W, et al. Functional connectivity of the retrosplenial cortex in rats with ischemic stroke is improved by electroacupuncture. Acupunct Med. 2020:964528420921190. doi:10.1177/0964528420921190.

23. Min YS, Park JW, Park E, Kim AR, Cha H, Gwak DW, et al. Interhemispheric Functional Connectivity in the Primary Motor Cortex Assessed by Resting-State Functional Magnetic Resonance Imaging Aids Long-Term Recovery Prediction among Subacute Stroke Patients with Severe Hand Weakness. J Clin Med. 2020;9(4). doi:10.3390/jcm9040975.

24. Chen J, Sun D, Shi Y, Jin W, Wang Y, Xi Q, et al. Altered static and dynamic voxel-mirrored homotopic connectivity in subacute stroke patients: a resting-state fMRI study. Brain Imaging Behav. 2020. doi:10.1007/s11682-020-00266-x.

25. Lu Q, Huang G, Chen L, Li W, Liang Z. Structural and functional reorganization following unilateral internal capsule infarction contribute to neurological function recovery. Neuroradiology. 2019;61(10):1181-90. doi:10.1007/s00234-019-02278-x.

26. Fragata I, Alves M, Papoila AL, Nunes AP, Ferreira P, Canto-Moreira N, et al. Early Prediction of Delayed Ischemia and Functional Outcome in Acute Subarachnoid Hemorrhage: Role of Diffusion Tensor Imaging. Stroke. 2017;48(8):2091-7. doi:10.1161/STROKEAHA.117.016811.

27. Luo $R$, Pan $P, X u Y$, Chen L. No reliable gray matter changes in essential tremor. Neurol Sci. 2019;40(10):2051-63. doi:10.1007/s10072-019-03933-0.

28. Gladstone DJ, Danells CJ, Black SE. The fugl-meyer assessment of motor recovery after stroke: a critical review of its measurement properties. Neurorehabil Neural Repair. 2002;16(3):232-40. doi:10.1177/154596802401105171.

29. Bushnell C, Bettger JP, Cockroft KM, Cramer SC, Edelen MO, Hanley D, et al. Chronic Stroke Outcome Measures for Motor Function Intervention Trials: Expert Panel Recommendations. Circ Cardiovasc Qual Outcomes. 2015;8(6 Suppl 3):163-9. doi:10.1161/CIRCOUTCOMES.115.002098.

30. Liu LB, Li M, Zhuo WY, Zhang YS, Xu AD. The role of hs-CRP, D-dimer and fibrinogen in differentiating etiological subtypes of ischemic stroke. PLoS One. 2015;10(2):e0118301. doi:10.1371/journal.pone.0118301.

31. Li J, Zhao X, Meng X, Lin J, Liu L, Wang C, et al. High-Sensitive C-Reactive Protein Predicts Recurrent Stroke and Poor Functional Outcome: Subanalysis of the Clopidogrel in High-Risk Patients With Acute Nondisabling Cerebrovascular Events Trial. Stroke. 2016;47(8):2025-30. doi:10.1161/STROKEAHA.116.012901.

32. Zhou Y, Han W, Gong D, Man C, Fan Y. Hs-CRP in stroke: A meta-analysis. Clin Chim Acta. 2016;453:21-7. doi:10.1016/j.cca.2015.11.027. 
33. Yoshimura S, Lindley RI, Carcel C, Sato S, Delcourt C, Wang X, et al. NIHSS cut point for predicting outcome in supra- vs infratentorial acute ischemic stroke. Neurology. 2018;91(18):e1695-e701. doi:10.1212/WNL.0000000000006437.

34. Chen CL, Chen CY, Chen HC, Wu CY, Lin KC, Hsieh YW, et al. Responsiveness and minimal clinically important difference of Modified Ashworth Scale in patients with stroke. Eur J Phys Rehabil Med. 2019;55(6):754-60. doi:10.23736/S1973-9087.19.05545-X.

35. Lee SY, Kim DY, Sohn MK, Lee J, Lee SG, Shin YI, et al. Determining the cut-off score for the Modified Barthel Index and the Modified Rankin Scale for assessment of functional independence and residual disability after stroke. PLoS One. 2020;15(1):e0226324. doi:10.1371/journal.pone.0226324.

36. Malmut L, Lin C, Srdanovic N, Kocherginsky M, Harvey RL, Prabhakaran S. Arm Subscore of Motricity Index to Predict Recovery of Upper Limb Dexterity in Patients With Acute Ischemic Stroke. Am J Phys Med Rehabil. 2020;99(4):300-4. doi:10.1097/PHM.0000000000001326.

37. Yuan H, Zhang N, Wang C, Luo BY, Shi Y, Li J, et al. Factors of Hamilton Depression Rating Scale (17 items) at 2 weeks correlated with poor outcome at 1 year in patients with ischemic stroke. Neurol Sci. 2014;35(2):171-7. doi:10.1007/s10072-013-1464-z.

38. Piersma D, Fuermaier ABM, de Waard D, De Deyn PP, Davidse RJ, de Groot J, et al. The MMSE should not be the sole indicator of fitness to drive in mild Alzheimer's dementia. Acta Neurol Belg. 2018;118(4):637-42. doi:10.1007/s13760-018-1036-3.

39. Jiang L, Geng H, Lu M, Du Z, Chen P, Han X, et al. Acupuncture for poststroke hemiplegia focusing on cerebral bilateral connections: study protocol for a randomised controlled neuroimaging trial. BMJ Open. 2020;10(4):e034548. doi:10.1136/bmjopen-2019-034548.

40. Poo MM, Du JL, Ip NY, Xiong ZQ, Xu B, Tan T. China Brain Project: Basic Neuroscience, Brain Diseases, and Brain-Inspired Computing. Neuron. 2016;92(3):591-6. doi:10.1016/j.neuron.2016.10.050.

41. Yang A, Wu HM, Tang JL, Xu L, Yang M, Liu GJ. Acupuncture for stroke rehabilitation. Cochrane Database Syst Rev. 2016;(8):CD004131. doi:10.1002/14651858.CD004131.pub3.

42. Fang J, Chen $L$, Ma R, Keeler $C L$, Shen $L$, Bao Y, et al. Comprehensive rehabilitation with integrative medicine for subacute stroke: A multicenter randomized controlled trial. Sci Rep. 2016;6:25850. doi:10.1038/srep25850.

43. Yang Y, Eisner I, Chen S, Wang S, Zhang F, Wang L. Neuroplasticity Changes on Human Motor Cortex Induced by Acupuncture Therapy: A Preliminary Study. Neural Plast. 2017;2017:4716792. doi:10.1155/2017/4716792.

44. Liu Z, Liu Y, Xu H, He L, Chen Y, Fu L, et al. Effect of Electroacupuncture on Urinary Leakage Among Women With Stress Urinary Incontinence: A Randomized Clinical Trial. JAMA. 2017;317(24):2493501. doi:10.1001/jama.2017.7220.

45. Rehme AK, Grefkes C. Cerebral network disorders after stroke: evidence from imaging-based connectivity analyses of active and resting brain states in humans. J Physiol. 2013;591(1):17-31. doi:10.1113/jphysiol.2012.243469. 
46. Xie Z, Cui F, Zou Y, Bai L. Acupuncture Enhances Effective Connectivity between Cerebellum and Primary Sensorimotor Cortex in Patients with Stable Recovery Stroke. Evid Based Complement Alternat Med. 2014;2014:603909. doi:10.1155/2014/603909.

47. Fu CH, Li KS, Ning YZ, Tan ZJ, Zhang Y, Liu HW, et al. Altered effective connectivity of resting state networks by acupuncture stimulation in stroke patients with left hemiplegia: A multivariate granger analysis. Medicine. 2017;96(47):e8897. doi:10.1097/MD.0000000000008897.

48. Han X, Bai L, Sun C, Niu X, Ning Y, Chen Z, et al. Acupuncture Enhances Communication between Cortices with Damaged White Matters in Poststroke Motor Impairment. Evid Based Complement Alternat Med. 2019;2019:4245753. doi:10.1155/2019/4245753.

49. Ning Y, Li K, Fu C, Ren Y, Zhang Y, Liu H, et al. Enhanced Functional Connectivity between the Bilateral Primary Motor Cortices after Acupuncture at Yanglingquan (GB34) in Right-Hemispheric Subcortical Stroke Patients: A Resting-State fMRI Study. Front Hum Neurosci. 2017;11:178. doi:10.3389/fnhum.2017.00178.

50. Zhang Y, Li KS, Ning YZ, Fu CH, Liu HW, Han X, et al. Altered structural and functional connectivity between the bilateral primary motor cortex in unilateral subcortical stroke: A multimodal magnetic resonance imaging study. Medicine. 2016;95(31):e4534. doi:10.1097/MD.0000000000004534.

\section{Figures}




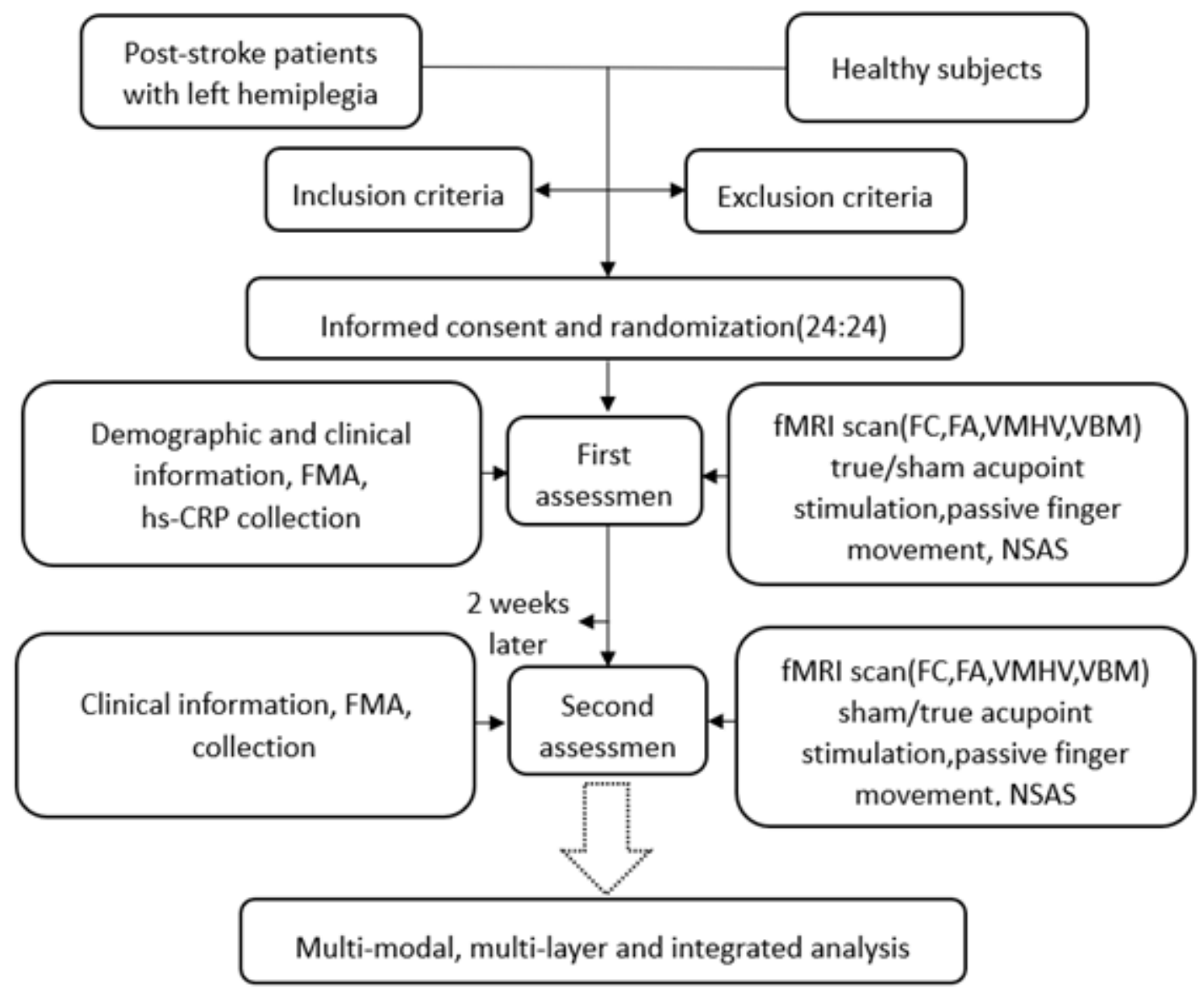

\section{Figure 1}

Flow chart of study design. 24:24, we plan to recruit 24 participants in patients' group and the healthy subjects' group respectively. FMA, Fugl-Meyer Assessment; hs-CRP, hypersensitive C-reactive protein; FC, functional connectivity; FA, fractional anisotropy; VMHC, Voxel-mirrored homotopic connectivity; VBM, Voxel-Based Morphometry; true acupoint, Yanglingquan(GB34); sham acupoint, located at 1 cun outward of Yanglingquan(GB34) horizontally; NSAS, Needle Sensation Assessment Scale 


\begin{tabular}{|c|c|c|c|c|}
\hline & \multicolumn{4}{|c|}{ STUDY PERIOD } \\
\hline \multirow[b]{2}{*}{\begin{tabular}{|l|} 
Time Point \\
\end{tabular}} & Enrolment & Allocation & \multicolumn{2}{|c|}{ Post-allocation } \\
\hline & week-1 & 0 & Baseline $(0)$ & Week 2 \\
\hline \multicolumn{5}{|l|}{ ENROLMENT } \\
\hline Eligibility Screen & $\mathrm{SP} / \mathrm{HS}$ & & & \\
\hline Informed Consent & $\mathrm{SP} / \mathrm{HS}$ & & & \\
\hline Randomization & & $\mathrm{SP} / \mathrm{HS}$ & & \\
\hline \multicolumn{5}{|c|}{ INTERVENTIONS(during fMRI scan) } \\
\hline $\begin{array}{l}\text { Acupoint } \\
\text { stimulation }\end{array}$ & & & $\mathrm{SP} / \mathrm{HS}$ & $\mathrm{SP} / \mathrm{HS}$ \\
\hline $\begin{array}{l}\text { Passive finger } \\
\text { movement }\end{array}$ & & & $\mathrm{SP} / \mathrm{HS}$ & $\mathrm{SP} / \mathrm{HS}$ \\
\hline \multicolumn{5}{|l|}{ ASSESSMENTS } \\
\hline fMRI Scan & & & $\mathrm{SP} / \mathrm{HS}$ & $\mathrm{SP} / \mathrm{HS}$ \\
\hline hs-CRP & & & SP & SP \\
\hline FMA & & & $\mathrm{SP}$ & SP \\
\hline NIHSS & & & $\mathrm{SP}$ & SP \\
\hline MAS & & & $\mathrm{SP}$ & SP \\
\hline BSS & & & SP & SP \\
\hline MRS & & & $\mathrm{SP}$ & SP \\
\hline $\mathrm{BI}$ & & & $\mathrm{SP}$ & SP \\
\hline MI & & & $\mathrm{SP}$ & SP \\
\hline HAMD & & & $\mathrm{SP} / \mathrm{HS}$ & $\mathrm{SP} / \mathrm{HS}$ \\
\hline MMSE & & & $\mathrm{SP} / \mathrm{HS}$ & $\mathrm{SP} / \mathrm{HS}$ \\
\hline NSAS & & & $\mathrm{SP} / \mathrm{HS}$ & $\mathrm{SP} / \mathrm{HS}$ \\
\hline Adverse Events & & & $\mathrm{SP} / \mathrm{HS}$ & $\mathrm{SP} / \mathrm{HS}$ \\
\hline
\end{tabular}

\section{Figure 2}

Schedule of enrollment, intervention, and assessment. SP, Stroke Patients; HS, Healthy Subjects; fMRI, functional magnetic resonance imaging; hs-CRP, high-sensitivity C-reactive protein; FMA, Fugl-Meyer Assessment; NIHSS, National Institutes of Health Stroke Scale; MAS, Modified Ashworth Scale; BSS, Brunnstrom Scale; MRS, Modified Rankin Scale; BI, Barthel Index; MI, Motricity Index; HAMD, Hamilton Depression Scale; MMSE, Mini-Mental State Examination; NSAS, Needle Sensation Assessment Scale 
rest in the supine position for 30

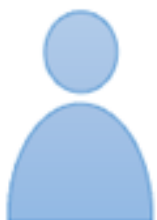
minutes; maintain the audiovisual closed state; the magnetic resonance coil will be fixed, and the head position will be kept still
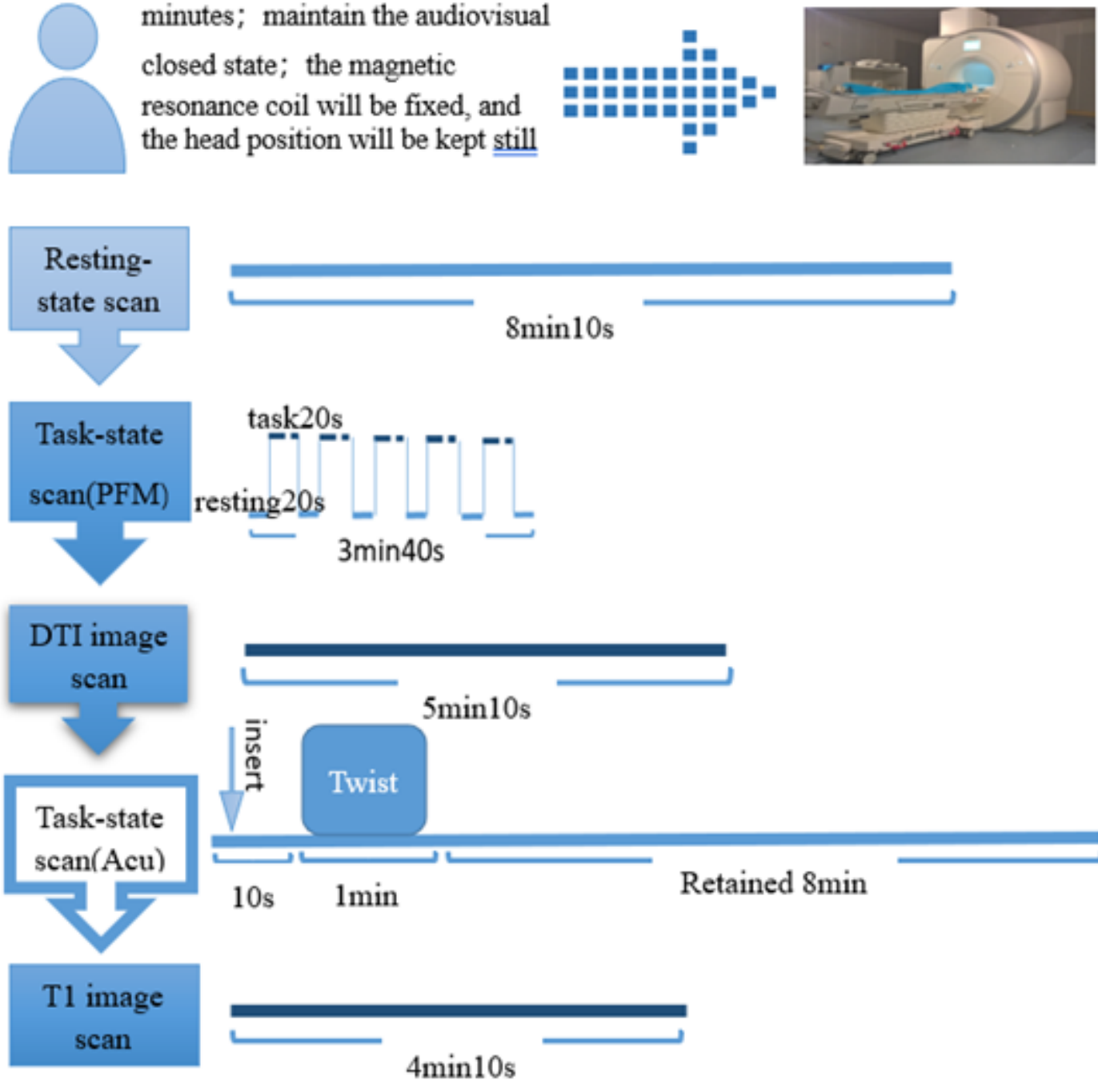

\section{Figure 3}

The detailed fMRI scheme. PFM, Passive Finger Movement; Acu, Acupuncture.

\section{Supplementary Files}

This is a list of supplementary files associated with this preprint. Click to download.

- Additionalfile1SPIRITchecklist.doc

- Additionalfile4Modelconsentformoriginalversion.pdf

- Additionalfile4Modelconsentformtranslation.pdf 